\title{
Expression patterns of CD168 correlate with the stage and grade of squamous cell carcinoma of head and neck
}

\author{
KORINNA JÖHRENS ${ }^{1}$, IOANNIS ANAGNOSTOPOULOS ${ }^{1}$, STEFFEN DOMMERICH ${ }^{2}$, JAN DIRK RAGUSE ${ }^{3}$, \\ AGNIESZKA J. SZCZEPEK ${ }^{2}$, FREDERICK KLAUSCHEN ${ }^{1}$ and KATHARINA STÖLZEL ${ }^{2}$ \\ ${ }^{1}$ Institute of Pathology, and ${ }^{2}$ Department of Otorhinolaryngology, Head and Neck Surgery, \\ Charité-Universitätsmedizin, Campus Charité Mitte, D-10117 Berlin; ${ }^{3}$ Department of Oral and Maxillofacial Surgery, \\ Charité-Universitätsmedizin, Campus Charité Virchow-Klinikum, D-13353 Berlin, Germany
}

Received August 8, 2016; Accepted February 13, 2017

DOI: $10.3892 / \mathrm{mco} .2017 .1165$

\begin{abstract}
The receptor for hyaluronan-mediated motility CD168 is associated with the processes of oncogenesis and metastasis. The objective of the present study was to determine the possible association between the expression and distribution of CD168 and the tumor stage of head-and-neck squamous cell carcinoma (SCC). Formalin-fixed and paraffinembedded tumor samples obtained from 100 patients during primary resection of SCC from the oral cavity, oropharynx, hypopharynx or larynx were included in the present study. The patients were divided into two risk groups: Low risk, representing the early stage of completely resected SCCs with good-to-moderate differentiation, and the high-risk group, representing the advanced stage SCCs with positive resection margins, vascular invasion or locoregional metastasis. All specimens were stained with a monoclonal antibody against CD168. Percentage and staining intensity of CD168-positive cells were scored, and their spatial distribution within the tumor nests was noted. The results obtained were correlated with the tumor stage. The quantification of CD168 expression revealed significant differences between the two risk groups (t-test, $\mathrm{P}=0.002$ ), with higher scores in tumors resected from the highrisk SSC group compared with those from the low-risk group. In addition, in the high-risk group, the CD168-positive cells were present predominantly in the periphery $(70.4 \%)$ of tumor nests, whereas in the low-risk group, only $56.6 \%$ were located there; however, this trend did not reach the level of statistical significance. Taken together, the results from the present study suggested that CD168 expression patterns could potentially be
\end{abstract}

Correspondence to: Dr Katharina Stölzel, Department of Otorhinolaryngology, Head and Neck Surgery, Charité-Universitätsmedizin, Campus Charité Mitte, Chariteplatz 1, D-10117 Berlin, Germany

E-mail: katharina.stoelzel@charite.de

Key words: CD168, squamous cell carcinoma of head and neck, low-risk tumors, high-risk tumors used as a predictor of tumor aggressiveness, and therefore they may be a prognostic factor in head-and-neck SCC.

\section{Introduction}

Head-and-neck cancers are among the six most common malignant tumors, and their incidence is still increasing (1). Squamous cell carcinomas (SCCs) comprise $90 \%$ of head-and-neck cancers (2). High consumption of alcohol and/or tobacco $(3,4)$ and viral infections [e.g., human papilloma virus (HPV)] $(5,6)$ represent two major risk factors for the development of head-and-neck SCC. Although the appropriate primary prevention measures, such as change of lifestyle or vaccination against HPV, already exist, not all cases of SCC can be prevented $(7,8)$. Therefore, an early detection of primary cancer and its recurrence remain the most important pillars of successful curative treatment.

To date, no immunohistochemical biomarkers predicting the clinical behavior of head-and-neck SCC have been described. Several reports $(9,10)$ have demonstrated that the overexpression of hypoxia-associated proteins, such as hypoxia-inducible factor- $\alpha$ and glucose transporter 1 , is associated with poor prognosis and resistance to radiotherapy in the oral SCCs (OSCCs). Similarly, the expression of transketolase-like protein 1 and the Apo10 protein epitope of the endonuclease, DNAseX, in OSCC in tissue or in peripheral blood has been successfully used for monitoring these tumors.

CD168, also known as a receptor for hyaluronic acidmediated motility (RHAMM), is a member of the hyaladherin family of hyaluronan-binding proteins. It is involved in cell signaling, migration, and adhesion via interactions with hyaluronan, microtubules, actin, calmodulin, and components of the extracellular regulated kinase (ERK) signaling pathway (11). Hyaluronan accumulates during high tissue turnover (e.g. in regeneration, wound healing, etc.), and is essential in cell migration and proliferation during embryogenesis (12). CD168 has been extensively studied in different tumor cell lines and a variety of tumor types, including lymphoid and myeloid malignancies, and colorectal, breast, ovarian and urothelial carcinoma (13-16), although only a few studies have dealt with its expression and significance in the head-and-neck SCCs (17-19). It has been demonstrated that CD168 is required 
to facilitate proliferation of head-and-neck cancer cells (18). In addition, CD168 was found to contribute to cell cycle abnormalities by co-expression with microtubule-associated protein homolog (TPX2), resulting in an increased proliferative activity of head-and-neck SCC. CD168 was found to be highly expressed in the clinical specimens of oropharynx, hypopharynx and laryngeal cancer (18). Notably, CD168 was recognized by cytotoxic $\mathrm{CD} 8^{+}$effector $\mathrm{T}$ cells, therefore having possible relevance in the development of an anti-SSC vaccine.

CD168 binds hyaluronan $(20,21)$ and together with another hyaluronan receptor, $\mathrm{CD} 44$, forms complexes that coordinately activate the mitogen-activated protein (MAP)/ERK1,2 pathway (22). CD168 is involved in the epithelial-to-mesenchymal transition (EMT) during the process of metastasis, and in the generation of breast cancer stem cells with enhanced resistance to therapy (19).

The present study aimed to characterize the CD168 expression pattern in primary SCC tissues resected from the oropharynx, hypopharynx, larynx and oral cavity. The total number of CD168-positive cells in tumor tissues were scored, and the spatial distribution pattern of CD168-positive tumor cells within the tumor cell nests (peripheral or central) were characterized, as well as the proportion of the positive tumor cells. The results obtained were analyzed in the context of clinical and pathological classification of the tumor-nodemalignant (TNM) tumors system for each patient, and within the risk groups.

\section{Patients and methods}

Patients and tissue specimens. The specimens were obtained from 100 patients diagnosed with the primary SCC of the oral cavity, oropharynx, hypopharynx or larynx. All patients included in the present study were admitted to the Charite University Hospital in Berlin. The study was approved by the local Ethics Committee (approval no. EA1/312/14).

In addition to the tumor localization, patients' gender, age and the TNM stage at diagnosis were evaluated. According to the Munich Tumor Registry, the clinical and pathological TNM stage represents significant prognostic factors independent of gender, age and HPV infection status (23). Therefore, our study sample was divided into two risk groups: Patients with early stage carcinomas (clinical/pathological T1 or clinical/pathological T2, N0, R0, L0, V0 stage) and a high-to-moderate histological differentiation (G1 and G2) were designated as the "low-risk" group, whereas patients with advanced stage cancer [clinical/pathological T3 or clinical/pathological T4, R0/1, L0/1, V0/1, N1-3, extracapsular spread (ESC)] and usually moderately to poorly differentiated carcinoma were assigned to the 'high-risk' group. ESC was defined according to the criteria of Woolgar and Triantafyllou (24). The lymph node specimens were separately screened by two pathologists for capsular destruction with or without tumor nests with desmoplastic reaction, as well as for focal ESC in the lymph node hilus. In difficult cases, serial sections were prepared and carefully examined.

The low-risk group consisted of 46 patients. In 5 of these patients ( 1 female and 4 males), the primary carcinoma was located in the oropharynx; in 2 (both males), the primary
Table I. Correlation between CD168 expression and the clinicopathological features $(n=100)$.

\begin{tabular}{lrrr}
\hline & \multicolumn{3}{c}{ CD168 expression } \\
\cline { 2 - 4 } Feature & $\mathrm{n}$ & Positive & Negative \\
\hline Age (years) & 1 & & \\
$20-39$ & 40 & 18 & 22 \\
$40-59$ & 55 & 16 & 39 \\
$60-79$ & 4 & & 4 \\
$80-99$ & & & \\
Gender & 16 & 6 & 10 \\
Female & 84 & 28 & 56 \\
Male & & & \\
Primary tumor site & 25 & 3 & 22 \\
Oral cavity & 22 & 12 & 10 \\
Oral pharynx & 13 & 9 & 4 \\
Hypopharynx & 40 & 10 & 30 \\
Larynx & 46 & 8 & 38 \\
Low-risk group & 54 & 26 & 28 \\
High-risk group & & & \\
\hline
\end{tabular}

carcinoma was located in the hypopharynx; in 17 patients (3 females and 14 males), it was located in the larynx carcinoma; and in the remaining 22 patients ( 7 females and 15 males), the primary carcinoma was located in the oral cavity. All the SCCs in these cases were highly or moderately differentiated (G1/G2). The high-risk group consisted of 54 patients. In 17 patients ( 2 females and 15 males), the tumor was located in the oropharynx; in 11 patients ( 1 female and 10 males), it was located in the hypopharynx; in 23 patients ( 2 females and 21 males), it was located in the larynx; and in the remaining 3 patients ( 1 female and 2 males), it was located in the oral cavity. The carcinomas had a variable differentiation grade (grade 1-3), but were predominantly G2.

Histology and immunohistochemistry. Formalin-fixed and paraffin-embedded tumor tissues were retrieved from the archives of the Pathology Institute at the Charité University Hospital in Berlin. Representative paraffin blocks encompassing vital tumor areas were selected. Each specimen was stained with hematoxylin and eosin for the histological tumor evaluation. Immunohistological analyses were performed using $4 \mu \mathrm{m}$ sections obtained from the selected paraffin blocks. To detect the CD168 protein (RHAMM), a mouse monoclonal antibody raised against human CD168 (clone 2D6, NCL-CD168; Leica Biosystems, Ltd., Newcastle, UK) was used at a dilution of 1:50. All immunostainings were performed with an automated immunostainer $\left(\mathrm{BondMax}^{\mathrm{TM}}\right.$; Leica Biosystems, Ltd.) according to the appropriate protocols and reagents for antigen retrieval and visualization, using a Bond Polymer Refine DAB Detection kit (Leica Biosystems, Ltd.). Depending on the size of specimens, 100-250 tumor cells were scored. The CD168-positive cells were noted as a percentage of all, positive and negative cells, and the staining intensities were evaluated. 
A

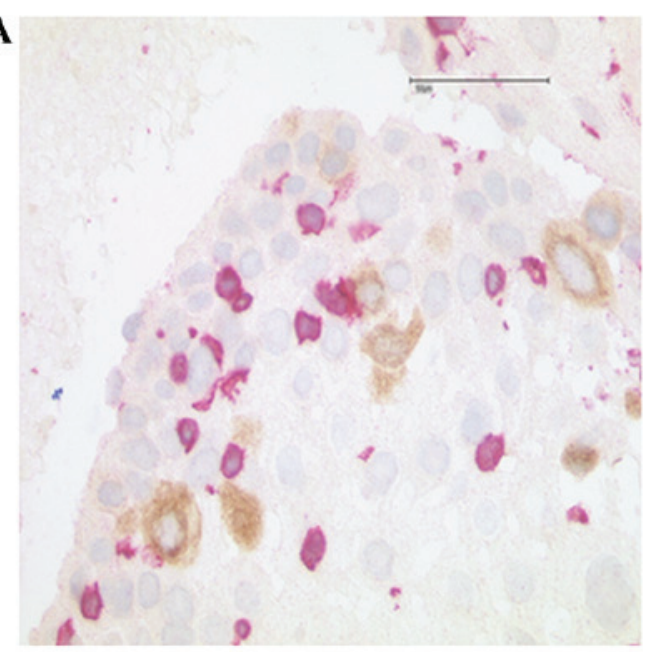

B

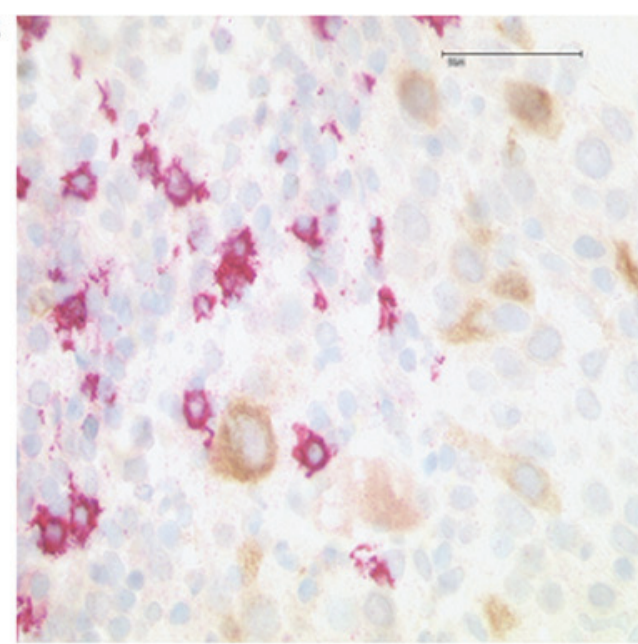

Figure 1. Verification of CD168-expressing cells. Double immunohistochemical staining experiments were performed for (A) CD3 and CD168 and (B) CD20 and CD168 proteins. The tumor-infiltrating T-cells (labeled with a monoclonal antibody against CD3; red immunostain) or B-cells (labeled with a monoclonal antibody against CD20; red immunostain) did not co-express CD168 (shown by the brown immunostain). The CD168-positive cells corresponded to neoplastic epithelial cells.

In order to verify CD168 expression by carcinoma cells, and not by tumor-infiltrating immune cells, a double immunostaining protocol was used, according to the protocol provided with the BondMax ${ }^{\mathrm{TM}}$ immunostainer (Leica Biosystems, Ltd.). CD168 expression was visualized using the Bond Polymer Refine DAB Detection kit (Leica Biosystems), resulting in a brown stain, whereas $\mathrm{CD} 20$ or $\mathrm{CD} 3$ were visualized with the Bond Polymer alkaline phosphatase kit, resulting in a red stain.

Statistical analysis. To quantitatively assess the immunohistochemical staining of CD168, the percentage of positive tumor cells and the staining intensity on a scale of $0,1.0,1.5,2.0,2.5$ and 3.0, corresponding to negative, weak, weak-to-moderate, moderate, moderate-to-strong and strong staining, respectively, were evaluated. The CD168 staining score was subsequently calculated as the product of positive tumor cells (as a percentage) and the intensity. The statistical significance of differences in the CD168 staining score between the low- and high-risk groups was calculated using the two-sided Student's t-test.

In addition to the continuous score described above, a cut-off of 20\% CD168-positive tumor cells was applied to dichotomize the cohort into CD168-positive and -negative tumors, and the results were correlated with the patients' age and localization of the primary tumor. The association between age and CD168 expression was analyzed by performing Pearson's correlation analysis. To compare the results regarding the spatial distribution of CD168-positive cells in the two risk groups, Pearson's Chi-square test was applied. Statistical calculations were performed using $\mathrm{R}$ software (version 3.2.0; The R Foundation for Statistical Computing: see https://www.r-project.org/). $\mathrm{P}<0.05$ was considered to indicate a statistically significant difference.

\section{Results}

Verification of CD168-expressing cells. Double immunohistochemical labeling revealed that the tumor-infiltrating
T- or B-cells did not express CD168, whereas all the CD168-positive cells corresponded to neoplastic epithelial cells (Fig. 1).

Quantification of CD168 expression. There was a significant difference in the CD168 staining scores for the low-risk [mean, 15.48; standard deviation (SD), 23.87] and high-risk (mean, 33.54; SD 31.72) groups [ $\mathrm{t}(95)=-3.141, \mathrm{P}=0.002]$. It was also observed that, in the high-risk group, more CD168-positive cells were localized to the periphery $(70.4 \%)$ compared with the low-risk group (56.6\%), although this trend was not statistically significant.

Using anti-CD168 staining with a positivity threshold arbitrarily set at $20 \%, 36 \%(n=36)$ of all 100 samples were identified as being CD168-positive. In patients with laryngeal cancer, there was a significant negative correlation between the age and CD168-positivity ( $r=-0.350 ; \mathrm{P}=0.027)$. Carcinomas located in the oropharynx and hypopharynx had a higher proportion of CD168-positive cases compared with the negative cases (ratio of 1.2 and 2.25 , respectively). The positivity of CD168 in the oral cavity carcinomas was low (ratio of 0.09) (Table I).

Localization of CD168 positive tumor cells in the low-risk group: Peripheral/central. In 20 of the total of 46 low-risk group cases (43.4\%), the CD168-positive tumor cells were localized either in the central tumor areas (11 cases), or no CD168 cells were detected (9 cases). In the remaining 26 cases $(56.6 \%)$, the CD168-positive tumor cells were detected at the tumor edge, with some cells located in the central areas (Fig. 2A).

Localization of CD168 positive tumor cells in the high-risk group: Peripheral/central. In 8 of the total of 54 high-risk group cases (14.8\%), the CD168-expressing tumor cells were localized in the central tumor areas, whereas 8 cases were CD168-negative. In the remaining 38 (70.4\%) cases, the CD168-positive cells were localized exclusively at the 
$\mathbf{A}$

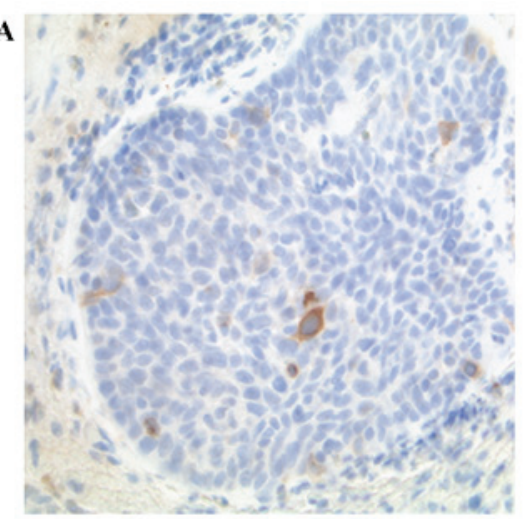

C

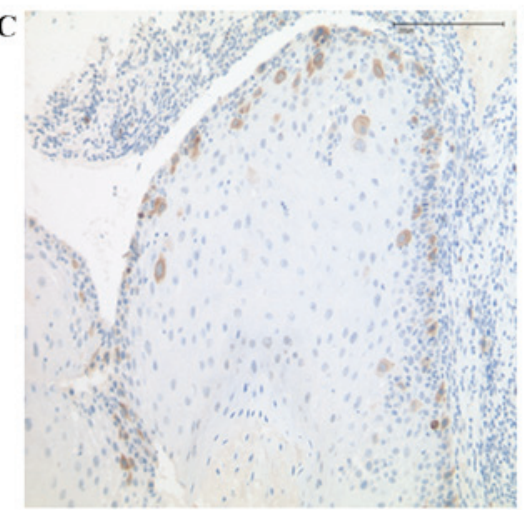

B
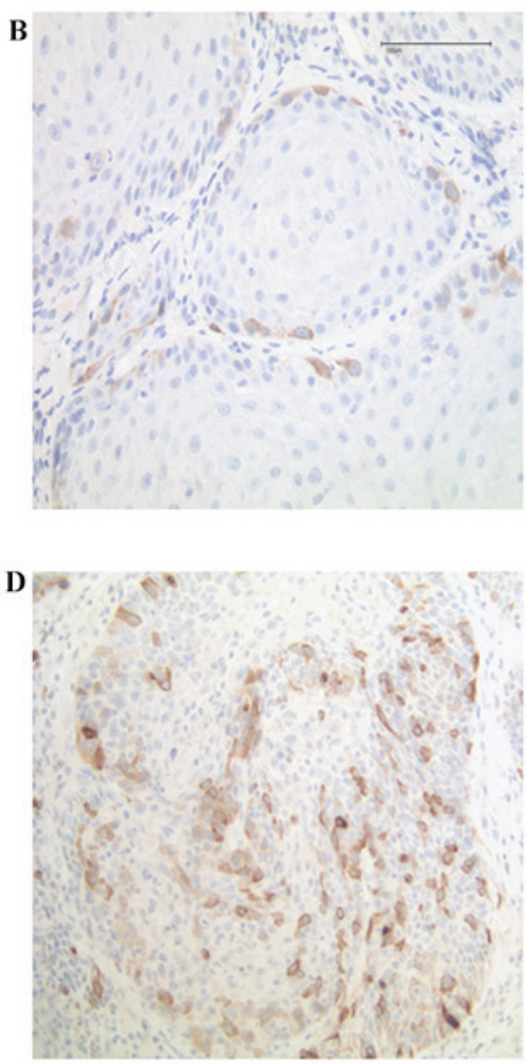

Figure 2. Representative examples of CD168 staining results. (A) Only a few tumor cells were CD168-positive, mainly in the central part of the tumor nest (low-risk tumors). (B and C) The CD168-positive cells accumulated at the edge of the tumor nest (high-risk tumors). (D) The CD168-positive cells were present at the edge and in the central parts of the tumor nest (high-risk tumors).

A

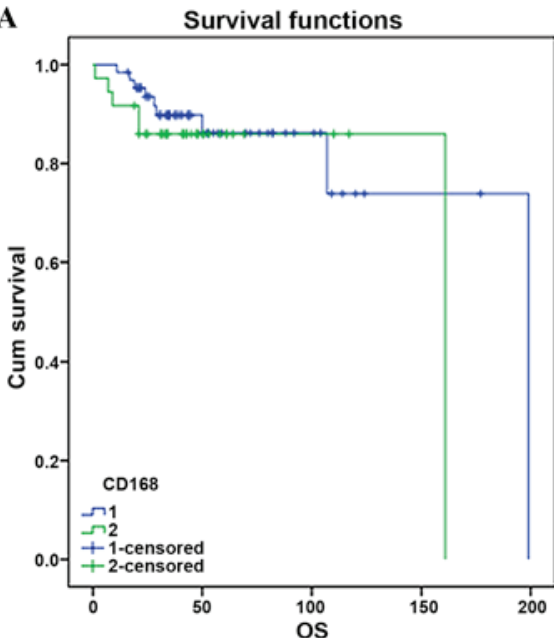

B

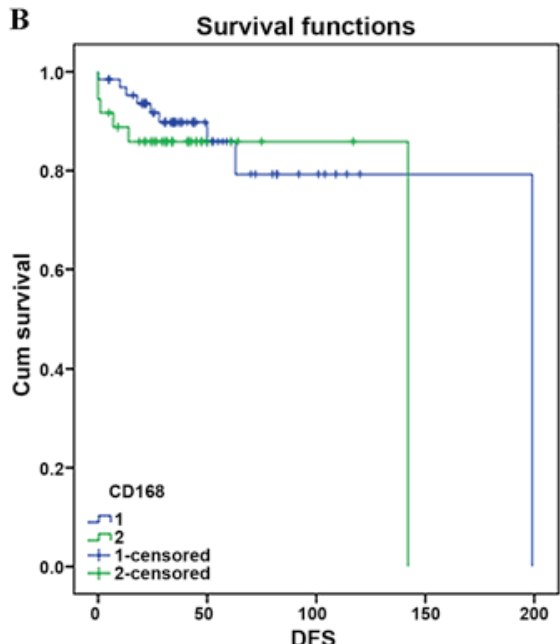

C

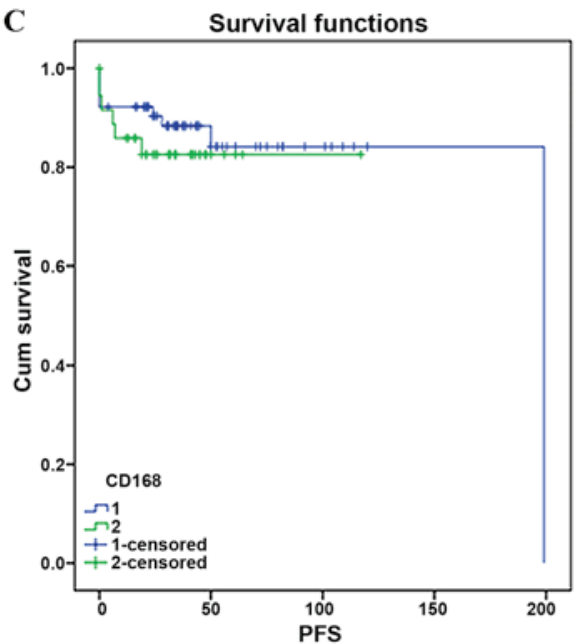

Figure 3. Kaplan-Meier-analysis for (A) OS, (B) DFS and (C) PFS, which did not reveal any significant differences for CD168 positive vs. negative cases (dichotomized at $20 \%$ CD168 positivity). OS, overall survival; DFS, disease-free survival; PFS, progression-free survival; Cum, cumulative.

tumor edge, or both at the edge and in the central tumor areas (Fig. 2B-D).

Kaplan-Meier analysis. Kaplan-Meier analysis was performed to determine overall survival, disease-free survival and progression-free survival. This analysis, however, did not reveal any significant differences for CD168-positive vs. -negative cases (dichotomized at 20\% CD168 positivity; Fig. 3).

\section{Discussion}

CD168, a RHAMM, has a dual function as an extracellular and an intracellular hyaluronan-binding-protein $(25,26)$. Hyaluronan serves an important role in tissue organization and in cellular migration, growth and differentiation (27). Several studies have highlighted a correlation between the expression of CD168 and cancer progression or a worse prognosis, particularly in the 
breast, stomach or colorectal cancers, and in multiple myelomas. A previous CD168 knockdown study demonstrated growth reduction of transplanted tumors (28). Increased CD168 expression was revealed to be indicative of an elevated, or abnormal, level of cell proliferation in human OSCC (17).

Several research groups subsequently turned their attention to the special subcellular localization of CD168. At the cell surface, CD168 mediates cell motility and growth, whereas within the cell, it is involved in the organization of the cytoskeletal network. Finally, when localized to the nucleus, CD168 functions as a regulator of cell cycle progression. In the present study, CD168 expression was observed predominantly in the cytoplasm, and a nuclear staining pattern was not detectable. The results of the present study corroborate those of Yamano et al (29), who investigated CD168 expression in oral SCC and determined a correlation not only with the TNM stage, but also with mRNA and protein expression, and also reported an exclusively cytoplasmic CD168 expression pattern. The novelty of the present study concerned an investigation of whether the topographic distribution of CD168-positive cells within the carcinoma nests correlated with the tumor stage.

The patients in the study were divided into two risk groups, according to the Munich tumor registry (23). This registry has evaluated the survival curves of head-and-neck cancer over the time period between 1998 and 2011, and the TNM stage was identified as a significant predictive factor (23). In the low-risk group, patients in the early stage of SCC (clinically/pathologically $\mathrm{T} 1$ or T2) were included, with total tumor resection (R0), without lymph node metastases, without vascular invasion and with good-to-moderate histological differentiation. The high-risk group included patients in the advanced stage of SSC (clinically/pathologically T3 or T4), with the infiltration of blood vessels or margins and with a variable differentiation grade. A highly significant statistical correlation was identified between the high-risk group and the presence of CD168-positive tumor cells in the present study, using the CD168 staining score. The data in the present study support the results of Yamano et al (29), who determined statistically significant higher expression levels of CD168 in the advanced stage of SCC (T3 and T4).

Since the expression of CD168 correlates positively with the migratory tumor potential (30), a further aim of the present study was to investigate the localization of CD168-positive tumor cells within the tumor tissues. It was identified that, indeed, there was a positive correlation between the presence of CD168-positive tumor cells at the edge of the tumor nests and the stage of cancer; however, this correlation did not reach the level of statistical significance.

Additionally, a significant negative correlation was identified between the patient's age and CD168 expression in the larynx carcinoma group. Studies revealing a similar correlation in other tumor types have delivered conflicting results: For example, in a breast cancer study, a positive correlation between the age and visceral metastases was identified (31). In another study on melanoma, the prognosis worsened with age, whereas the number of CD168-positive sentinel nodes decreased (32). The authors of that study suggested that hematogenous tumor spread may account for this paradox (32).

Kaplan-Meier analysis for overall survival, disease-free survival and progression free-survival was performed, which did not reveal any significant differences for CD168 positive vs. negative cases. It is worth noting that our survival analysis has (at present) very limited utility due to the short observation time. The clinical course of the patients will continue to be recorded and assessed, and the survival analysis will be performed again at a certain time in the future.

In conclusion, using a representative group of head-andneck SCC specimens, it has been demonstrated, to the best of our knowledge for the first time, that an increased number of CD168-positive cells correlates with tumor grade and the TNM stage. Consequently, CD168 may be used as a possible prognosis marker. The present authors suggest that a routine evaluation of the CD168 expression pattern in cases of head-and-neck SCC may be helpful in evaluating the tumor aggressiveness, and in predicting individual patient prognosis.

\section{References}

1. Jemal A, Bray F, Center MM, Ferlay J, Ward E and Forman D: Global cancer statistics. CA Cancer J Clin 61: 69-90, 2011.

2. Gao J, Panizza B, Johnson NW, Coman S and Clough AR: Basic consideration of research strategies for head and neck cancer. Front Med 6: 339-353, 2012.

3. Gangane N, Chawla S, Anshu, Gupta SS and Sharma SM: Reassessment of risk factors for oral cancer. Asian Pac J Cancer Prev 8: 243-248, 2007.

4. Accortt NA, Waterbor JW, Beall C and Howard G: Cancer incidence among a cohort of smokeless tobacco users (United States). Cancer Causes Control 16: 1107-1115, 2005.

5. Paz IB, Cook N, Odom-Maryon T, Xie Y and Wilczynski SP: Human papillomavirus (HPV) in head and neck cancer. An association of HPV 16 with squamous cell carcinoma of Waldeyer's tonsillar ring. Cancer 79: 595-604, 1997.

6. Kreimer AR, Clifford GM, Boyle P and Franceschi S: Human papillomavirus types in head and neck squamous cell carcinomas worldwide: A systematic review. Cancer Epidemiol Biomarkers Prev 14: 467-475, 2005.

7. Zaravinos A: An updated overview of HPV-associated head and neck carcinomas. Oncotarget 5: 3956-3969, 2014.

8. Saraiya M, Unger ER, Thompson TD, Lynch CF, Hernandez BY, Lyu CW, Steinau M, Watson M, Wilkinson EJ, Hopenhayn C, et al; HPV Typing of Cancers Workgroup: US assessment of HPV types in cancers: Implications for current and 9-valent HPV vaccines. J Natl Cancer Inst 107: djv086, 2015.

9. Grimm M, Schmitt S, Teriete P, Biegner T, Stenzl A, Hennenlotter J, Muhs HJ, Munz A, Nadtotschi T, König K, et al: A biomarker based detection and characterization of carcinomas exploiting two fundamental biophysical mechanisms in mammalian cells. BMC Cancer 13: 569, 2013.

10. Grimm M, Cetindis M, Lehmann M, Biegner T, Munz A, Teriete P and Reinert S: Apoptosis resistance-related ABCB5 and DNaseX (Apo10) expression in oral carcinogenesis. Acta Odontol Scand 73: 336-342, 2015.

11. Lynn BD, Li X, Cattini PA, Turley EA and Nagy JI: Identification of sequence, protein isoforms, and distribution of the hyaluronan-binding protein RHAMM in adult and developing rat brain. J Comp Neurol 439: 315-330, 2001.

12. Choudhary M, Zhang X, Stojkovic P, Hyslop L, Anyfantis G, Herbert M, Murdoch AP, Stojkovic M and Lako M: Putative role of hyaluronan and its related genes, HAS2 and RHAMM, in human early preimplantation embryogenesis and embryonic stem cell characterization. Stem Cells 25: 3045-3057, 2007.

13. Karamitopoulou E, Zlobec I, Panayiotides I, Patsouris ES, Peros G, Rallis G, Lapas C, Karakitsos P, Terracciano LM and Lugli A: Systematic analysis of proteins from different signaling pathways in the tumor center and the invasive front of colorectal cancer. Hum Pathol 42: 1888-1896, 2011.

14. Knüpfer MM, Poppenborg H, Hotfilder M, Kühnel K, Wolff JE and Domula M: CD44 expression and hyaluronic acid binding of malignant glioma cells. Clin Exp Metastasis 17: 71-76, 1999.

15. Assmann V, Gillett CE, Poulsom R, Ryder K, Hart IR and Hanby AM: The pattern of expression of the microtubule-binding protein RHAMM/IHABP in mammary carcinoma suggests a role in the invasive behaviour of tumour cells. J Pathol 195: 191-196, 2001. 
16. Kong QY, Liu J, Chen XY, Wang XW, Sun Y and Li H: Differential expression patterns of hyaluronan receptors CD44 and RHAMM in transitional cell carcinomas of urinary bladder. Oncol Rep 10: 51-55, 2003.

17. Schmitt A, Barth TF, Beyer E, Borchert F, Rojewski M, Chen J, Guillaume P, Gronau S, Greiner J, Möller P, et al: The tumor antigens RHAMM and G250/CAIX are expressed in head and neck squamous cell carcinomas and elicit specific CD8+ T cell responses. Int J Oncol 34: 629-639, 2009.

18. Shigeishi H, Fujimoto S, Hiraoka M, Ono S, Taki M, Ohta K, Higashikawa $\mathrm{K}$ and Kamata N: Overexpression of the receptor for hyaluronan-mediated motility, correlates with expression of microtubule-associated protein in human oral squamous cell carcinomas. Int J Oncol 34: 1565-1571, 2009.

19. Shigeishi H, Biddle A, Gammon L, Emich H, Rodini CO Gemenetzidis E, Fazil B, Sugiyama M, Kamata N and Mackenzie IC: Maintenance of stem cell self-renewal in head and neck cancers requires actions of GSK3 $\beta$ influenced by CD 44 and RHAMM. Stem Cells 31: 2073-2083, 2013.

20. Turley EA, Noble PW and Bourguignon LY: Signaling properties of hyaluronan receptors. J Biol Chem 277: 4589-4592, 2002.

21. Wang C, Entwistle J, Hou G, Li Q and Turley EA: The characterization of a human RHAMM cDNA: Conservation of the hyaluronan-binding domains. Gene 174: 299-306, 1996.

22. Telmer PG, Tolg C, McCarthy JB and Turley EA: How does a protein with dual mitotic spindle and extracellular matrix receptor functions affect tumor susceptibility and progression? Commun Integr Biol 4: 182-185, 2011.

23. Schlesinger-Raab AHU, Schubert-Fritschle G and Engel J: Epidemiologie der Kopf-Hals-Tumoren. In: Kopf-HalsMalignome Mast G, München, 2014.

24. Woolgar JA and Triantafyllou A: Pitfalls and procedures in the histopathological diagnosis of oral and oropharyngeal squamous cell carcinoma and a review of the role of pathology in prognosis. Oral Oncol 45: 361-385, 2009.
25. Turley EA, Hossain MZ, Sorokan T, Jordan LM and Nagy JI: Astrocyte and microglial motility in vitro is functionally dependent on the hyaluronan receptor RHAMM. Glia 12: 68-80, 1994.

26. Turley EA, Austen L, Moore D and Hoare K: Ras-transformed cells express both CD44 and RHAMM hyaluronan receptors: Only RHAMM is essential for hyaluronan-promoted locomotion. Exp Cell Res 207: 277-282, 1993

27. Laurent TC: Biochemistry of hyaluronan. Acta Otolaryngol Suppl 442: 7-24, 1987.

28. Niedworok C, Kretschmer I, Röck K, Vom Dorp F, Szarvas T, Hess J, Freudenberger T, Melchior-Becker A, Rübben H and Fischer JW: The impact of the receptor of hyaluronan-mediated motility (RHAMM) on human urothelial transitional cell cancer of the bladder. PLoS One 8: e75681, 2013.

29. Yamano Y, Uzawa K, Shinozuka K, Fushimi K, Ishigami T, Nomura H, Ogawara K, Shiiba M, Yokoe H and Tanzawa H: Hyaluronan-mediated motility: A target in oral squamous cell carcinoma. Int J Oncol 32: 1001-1009, 2008.

30. Akiyama Y, Jung S, Salhia B, Lee S, Hubbard S, Taylor M, Mainprize T, Akaishi K, van Furth W and Rutka JT: Hyaluronate receptors mediating glioma cell migration and proliferation. $\mathrm{J}$ Neurooncol 53: 115-127, 2001.

31. Rosa Mendoza ES, Moreno E and Caguioa PB: Predictors of early distant metastasis in women with breast cancer. J Cancer Res Clin Oncol 139: 645-652, 2013.

32. Page AJ,Li A,Hestley A, Murray D, Carlson GW and Delman KA: Increasing Age Is Associated with Worse Prognostic Factors and Increased Distant Recurrences despite Fewer Sentinel Lymph Node Positives in Melanoma. Int J Surg Oncol 2012: 456987, 2012. 